\title{
The effects of local and global link creation mechanisms on contagion processes unfolding on time-varying networks
}

\author{
Kaiyuan Sun, Enrico Ubaldi, Jie Zhang, Márton Karsai and Nicola Perra
}

\begin{abstract}
Social closeness and popularity are key ingredients that shape the emergence and evolution of social connections over time. Social closeness captures local reinforcement mechanisms which are behind the formation of strong ties and communities. Popularity, on the other hand, describes global link formation dynamics which drive, among other things, hubs, weak ties and bridges between groups. In this chapter, we characterize how these mechanisms affect spreading processes taking place on time-varying networks. We study contagion phenomena unfolding on a family of artificial temporal networks. In particular, we revise four different variations of activity-driven networks that capture i) heterogeneity of activation patterns ii) popularity iii) the emergence of strong and weak ties iv) community structure. By means of analytical and numerical analyses we uncover a rich and process dependent phenomenology where the interplay between spreading phenomena and link formation mechanisms might either speed up or slow down the spreading.
\end{abstract}

Key words: Activity driven networks, epidemic modeling, dynamical processes on time-varying networks, time-varying networks models, popularity, social closeness

Kaiyuan Sun

MOBS Lab, Network Science Institute, Northeastern University, Boston, USA e-mail: contactsunky@gmail.com

Enrico Ubaldi

Sony Computer Science Laboratories, Paris, France e-mail: enricoubaldi@gmail.com

Jie Zhang

Networks and Urban Systems Centre, University of Greenwich, London, UK e-mail: jie.zhang@gre.ac.uk

Márton Karsai

Univ Lyon, Inria, CNRS, ENS de Lyon, UCB Lyon 1, LIP UMR 5668, F-69007 Lyon, France email: marton.karsai@ens-lyon.fr

Nicola Perra

Networks and Urban Systems Centre, University of Greenwich, London, UK e-mail: n.perra@gre.ac.uk 


\section{Introduction}

Think about the last conference you attended. In particular, focus on the social interactions you had throughout the week. Chances are that you spent a disproportionate fraction of time chatting with old and current collaborators as well as with long time colleagues you typically meet in such settings. Chances are that you also networked with new people. Most of these interactions were probably short and spontaneous exchanges maybe while waiting in line for coffee or after your presentation. Others might have been with editors, to whom you were trying to push your new idea for a book, or with one of the keynote speakers after her inspiring talk. Bear with us and think about last time you visited Twitter. Chances are that you read and interacted with the posts of popular users, which you don't know personally, but follow avidly. Finally, chances are that you also interacted with your personal friends you follow.

These two scenarios highlight how both face-to-face and digital interactions are temporal acts driven by intricate social mechanisms. Among them we can identify two categories. The first, refers to frequent reciprocated connections with individuals in your close social circle(s). These are interactions you reinforce and activate over time. The second refers to (mostly) one-sided interactions with popular individuals. These are connections you initiate with people who, thanks to their status and fame, are able to attract a large share of the attention from many others. The first category encompasses local mechanisms that do not depend on the behavior of people outside your social circle. The second instead, global mechanisms that depend on the behavior of, potentially, a large fraction of individuals.

Let's go back to your last conference. Many of the interactions you probably had were with people that, conference after conference, dinner after dinner, paper after paper, entered in your close social circle. These are individuals that you know very well and that are likely part of the same community. Some of the other interactions instead were probably with popular and influential people such as the keynote speakers or editors with whom many other participants wanted to speak to. The first type of interactions were driven by social closeness, the second instead by popularity.

A large literature, mostly built on a time-aggregated (static) data, substantiates this picture. In particular, it is well known that social ties (both offline and online) can be categorized as strong or weak $[1,2,3,4,5,6,7,8]$. The first describes a small subset of ties which are activated frequently. The second instead describes sporadic (such as the person you met while waiting for coffee) interactions. A classic signature of this tendency is found in the distributions of link's weights which are heterogeneous. There is more. In fact, as alluded above, people with whom we share strong ties are likely to be also connected in tight communities [3, 9, 10, 11]. Thus, strong ties are clustered around groups of people characterized by large links' overlap $[3,12]$. Some of the weak ties instead, bridge such groups [3, 13]. Another well known property of real networks is the heterogeneity in the distribution of number of ties (the degree) $[14,15]$. In fact, networks are typically sparse. Many nodes are 
poorly connected. Few of them instead are able to attract a disproportionate amount of connections. It is important to stress how strong and weak ties, communities, and hubs emerge and evolve over time $[16,17]$. Which model(s) can be used to reproduce such features? How do they affect contagion phenomena taking place on their fabric? These are the main questions we will tackle in this chapter. In particular, we aim to revise and discuss a set of models that have been proposed to capture the evolution of social ties as function of time. In particular, we will consider both local and global approaches able to reproduce the formation of strong ties, communities, as well as the presence of popular individuals. From this stand point, we will then study how they affect contagion (epidemic) processes unfolding on such networks.

\section{The activity-driven framework}

To explore the effects of local and global link formation mechanisms on epidemic spreading processes, we will consider several variations of the activity-driven framework [18]. These are models of time-varying networks, based on the same fundamental scheme. In particular, when one is tasked to describe the evolution of the connections between $N$ nodes, needs to specify (at least) which nodes are involved in interactions at each time step. In the activity-driven framework this prescription is divided in two steps: i) activation, ii) partners selection. The first describes, which nodes are active and willing to connect. The second instead describes how such active nodes select the partners to whom interact. The modeling of the activation process, which will be the same for all the different variations of the framework we discuss here, is based on the intuition that not all nodes are equally willing to create or be part of social interactions. This has been confirmed with a range of observations in real datasets capturing very different type of interactions ranging from scientific collaborations to R\&D alliances between firms [18, 19, 20, 21, 22, 23, 24]. In particular, it turns out that the activity rate (measured in series of time windows of size $\Delta t$ ) is very heterogeneous. Furthermore, the distribution of activity is largely independent on the choice of $\Delta t$. In other words, if we measure the propensity of nodes to be involved in a social interaction by splitting the data in time windows of size $\Delta t$ or $\Delta t^{\prime}$ we will get very extremely similar distributions. The partner selection process instead describes the mechanism behind the formation of links. Here, we will revise and consider three different models that capture popularity and social closeness mentioned above. In addition, we will consider a basic version of the model in which link creation is random. This will serve as null model (baseline). In all cases, we will first discuss the details of the link formation mechanism and then their effect on epidemic processes unfolding on the network at comparable time scale respect to the evolution on the graph's structure.

The general setting of activity driven models is the following. $N$ nodes are described by at least one variable: their activity $a$. This quantity regulates their propen- 
sity to be active and willing to initiate social interactions at each time step. Activities are extracted and assigned to nodes from a distribution $F(a)$, which in the following we consider as a power-law. Thus $F(a)=C a^{-\gamma}$ with $\varepsilon \leq a \leq 1$ to avoid divergences for small values of activities. At each time step $t$ the network $G_{t}$ starts completely disconnected. Each node $i$ is active with a probability $a_{i} \Delta t$. Active nodes create $m$ connections with others. As mentioned above we will consider four link creation mechanisms. At time $t+\Delta t$ each link is deleted and the process re-start and a network $G_{t+\Delta t}$ is generated. It is important to stress how all the links are deleted at the end of each iteration and thus links do not persist across time steps unless they are re-formed.

\subsection{Model 1: baseline}

The simplest link formation mechanism is random [18] (see Figure 1-A). In this very unrealistic scenario partners are selected homogeneously across the entire system. While, very active nodes are likely to initiate connections in adjacent time steps, the probability that the same link is activated more than one time, the weight in a time integrated network, follows a Poisson distribution which, as mentioned in the introduction, is quite far from real observations. However, it is possible to show that integrating links over $T$ time steps, the degree distribution follows the functional form of the activity [38]. Thus, the heterogeneous propensity to initiate social interactions results in a heterogeneous degree distribution. It is important to stress however, that each $G_{t}$ network is mostly constituted by a set of disconnected stars formed around active nodes. Hubs emerge in time due the active engagement of such nodes.

\subsection{Model 2: global links formation process driven by popularity}

The second link formation we consider aims to capture a global, popularity based, mechanism [23] (see Figure 1-B). The basic intuition here is that not all nodes are equally attractive. Keynotes in conferences and celebrities in Twitter attract a disproportionate fraction of the connections in the system. To account for this, we assume that nodes, besides the activity, are characterized by another feature: the attractiveness $b$. Observations in different online social platforms suggest that indeed the propensity of people to attract connections is heterogeneously distributed [23]. All these aspects can be modeled within the activity-driven framework as follows. Nodes are assigned with two features: activity $a$ and attractiveness $b$. These two quantities are extracted from a joint distribution $H(a, b)$. Interestingly, observations on online social platforms indicate that active people are also more attractive, thus 
the two features are in general positively correlated [23]. In these settings the dynamics of the networks are very similar as those described above. At each time step $t$, the network $G_{t}$ starts disconnected. Each node is active with probability $a \Delta t$ and connects to $m$ other nodes. Each partner $j$ is selected following a simple preferential attachment, thus with probability $b_{j} /\langle b\rangle N$. At time $t+\Delta t$ all links are deleted and the process starts from scratch. It is possible to show that also in this case the degree distribution, obtained integrating links over time, is heterogeneous. However, the presence of attractiveness introduces some levels of heterogeneity in the weight distribution [23].

\subsection{Model 3: local links formation process driven by social memory}

The third link formation mechanism is local and based on the idea of social closeness (see Figure 1-C). The intuition is that, due to the need for close social connection, cognitive and temporal constraints, large part of our interactions take place within a small social circle $[4,25,26,27,28,29,30]$. These are strong ties that we remember (hence social memory) and activate frequently. We do also have sporadic, weak, interactions with people outside the circle such as the conference participants we met waiting for coffee in the hypothetical scenario described in the introduction. Observations across collaboration and communication networks corroborate this picture $[3,13,20,22,23,24]$. Indeed, the probability that the next social act, for nodes that have already contacted $k$ distinct individuals in the past, will result in the establishment of a new, $k+1$-th, tie follows this function $p(k)=\left(1+\frac{k}{c}\right)^{-\eta}[20,22]$. This implies that the larger the size of social circle the smaller the probability of increasing it. Consequently, social acts are frequently repeated within small circles of nodes. Remarkably, the behavior of large number of individuals can be modeled using one single value of $c$ (that captures the off-set after which the memory effects become effective) and a single (or multiple within a small region) value of $\eta$ (that captures the memory strength) $[20,22]$. We can modify the activity-driven framework to account for the function $p(k)$ that regulates the tendency towards new/old connections (social memory). The evolution of these networks, driven by such local link formation mechanism, is modeled as follows. At each time $t$ the network $G_{t}$ starts completely disconnected. Each node $i$ activates with probability $a_{i} \Delta t$ and creates $m$ connections. Each of these is created towards a randomly selected node never contacted before with probability $p\left(k_{i}\right)$ (where $k_{i}$ is the number of nodes already in the social circle of $i$ ) and with probability $1-p\left(k_{i}\right)$ towards a node already connected before. It is possible to show how both emergent degree and weight distributions are heterogeneous and function of $\eta[20,22]$. 


\subsection{Model 4: local links formation process driven by communities}

The fourth, and final, mechanism is also based on a local link formation process. As mentioned in the introduction, not only our social ties are organized around strong and weak ties but people in our social circles are likely to be friends. In networks terms, social circles are communities formed by groups of tightly connected people [9]. It is important to notice how the local mechanism described above (model 3) does not account for this important aspect. Indeed, triangles (i.e. a friend of my friend is my friend), which are crucial aspects of real communities, might emerge but are not likely by construction. There has been multiple proposals on how to model emergent clusters using the activity-driven framework [31, 32]. Here, we will consider the approach developed in Ref. [32] (see Figure 1-D). Observations in real networks show how the size of communities is heterogeneous [9]. Thus, we can set a distribution $P(s) \sim s^{-\alpha}$ with $s_{\text {min }} \leq s \leq s_{\max }$ to describe the size, $s$, of communities in the system. Each node is then associated to a community. In these settings, we can easily modify the activity-driven framework to account for communities. As before, at each time step $t$ the network $G_{t}$ starts completely disconnected. Each node $i$ is active with probability $a_{i} \Delta t$ and creates $m$ connections. With probability $q$ each of these is directed towards nodes in the same community (at random). With probability $1-q$ instead links are created randomly outside the community. The parameter $q$ regulates the modularity of the time-aggregated network. Clearly, if $q=0$ the community structure does not play any role on the dynamics of the network. Instead, if $q=1$ the system is formed by disconnected communities. Values in between connect these two limits. It is possible to show how, for moderately high values of $q$, the degree and weight distribution are heterogeneous [32].

\section{Epidemic spreading on activity-driven networks: analytical approach}

After this preamble, we are in the position to investigate how global and local link formation mechanisms affect epidemic spreading. To this end, we will consider a prototypical contagion process: the Susceptible-Infected-Susceptible model [33, 34, 35]. Here, nodes are divided in two compartments according to their disease status. Susceptible, $S$ for short, are healthy nodes that might be infected. Infected, $I$ for short, are infectious nodes. The natural history of the disease is described by two transitions. The first is the infection process which is linked to a contact between $S$ and $I$ nodes. In particular, a susceptible node in contact with an infected one gets infected with probability $\lambda: S+I \stackrel{\lambda}{\rightarrow} 2 I$. The second is instead the recovery process. Infected nodes spontaneously recover and become susceptible again with rate $\mu: I \stackrel{\mu}{\rightarrow} S$. A key quantity, that can be used to study the spreading of a disease with a given $\lambda$ and $\mu$ in a given network, is the epidemic threshold. The 


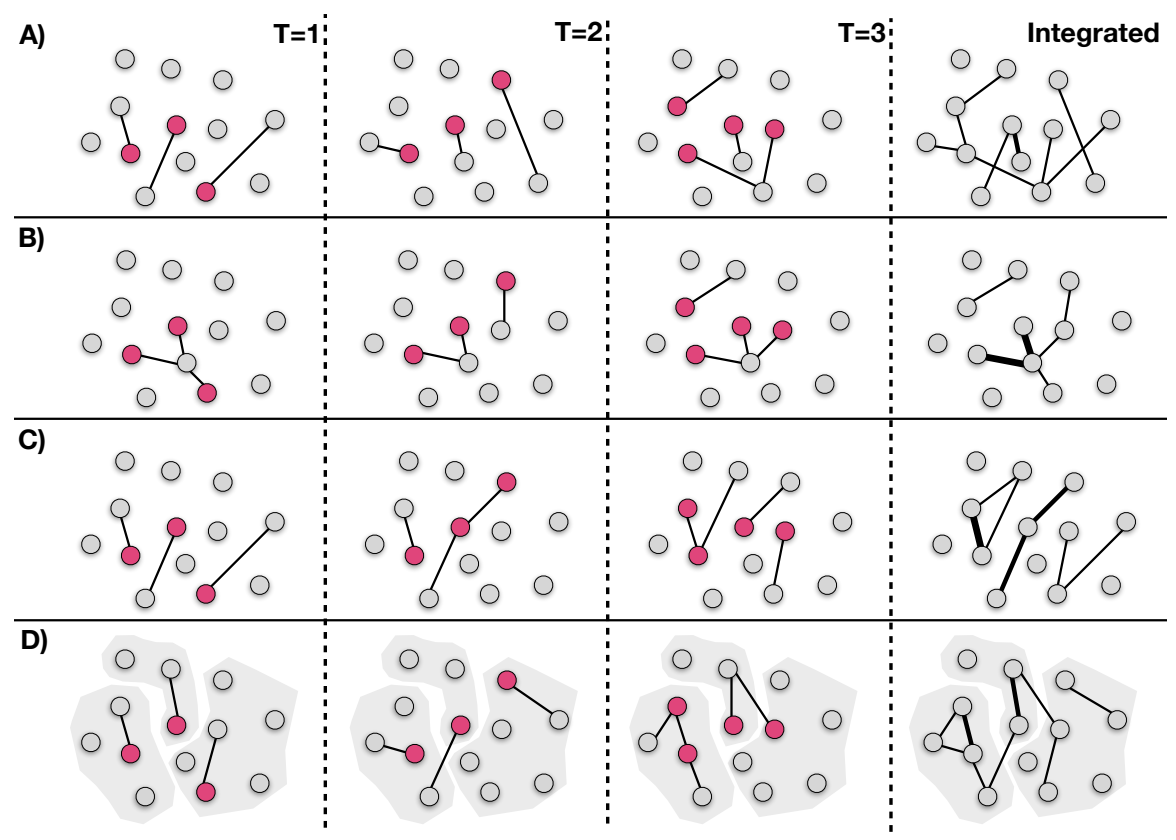

Fig. 1 Schematic representation of the four different variations of the activity-driven framework. The first three columns describe three time-steps of the evolution of network. The final column describes the union of links created in the three time steps. At each time, for all the networks, we assume that the same nodes are active (nodes in red), but the link creation process is instead different. For simplicity we assume $m=1$, the width of the link in the final column is proportional to how many time each link was activated. The first row (A) shows the case of random link creation (model 1). The second (B) describe the global link creation mechanism based on popularity (model 2). In this representation, one of nodes (the node with degree 4 in the integrated network) is the most attractive node. The third row (C) describes the local link creation mechanism based on the social memory (model 3). The final row (D) describes the local link creation mechanism driven by the presence of communities which are depicted by shaded areas (model 4).

disease will be able to spread, and reach an endemic state, only above a critical value (which is determined by the features of the network where the process unfolds). Below such critical value the disease will die out and affect only a small fraction of the population. Before going forward we need another piece. In particular, how do we go about estimating, numerically, the epidemic threshold? The classic approach is to study $I_{\infty}$ as function of $\lambda / \mu[34,36]$. As already mentioned, above the threshold the process reaches an endemic state. This is a dynamical equilibrium in which the total number of infected nodes is constant. Thus above threshold $I_{\infty}>0$, while below threshold $I_{\infty}=0$. Due to the stochastic nature of the process, the estimation of the threshold by looking at the behavior of $I_{\infty}$ is quite hard. We will adopt an alternative and recent approach, which looks at the life time, $L$, of the process [37]. This quantity is defined as the time the disease needs either to die out or to reach a 
finite fraction, $Y$, of the population. Indeed, well below the threshold the disease will quickly die out. Well above the threshold the disease will be able to reach the fraction $Y$ quite quickly. For values between these two regimes the life time increases and reaches a peak for values in proximity of the real threshold. In the language of phase transitions, the life time acts as the susceptibility $\chi$ [37].

We have now all the ingredients to study a contagion process unfolding on different versions of activity-driven networks which capture local and global link formation dynamics. Before jumping to the details we summarize the key features of the various models in Table 1 .

\begin{tabular}{|l|l|l|}
\hline Model & Links creation mechanism & Key variable(s) \\
\hline 1 & Random & Activity $a$ \\
\hline 2 & Global: driven by popularity & Activity $a$ and attractiveness $b$ \\
\hline 3 & Local: driven by social memory & $\begin{array}{l}\text { Activity } a \text {, social ties reinforcement parameters } c \\
\text { and } \eta\end{array}$ \\
\hline 4 & Local: driven by communities & $\begin{array}{l}\text { Activity } a \text {, details of communities sizes distribu- } \\
\text { tion, probability } q \text { to select partners in the same } \\
\text { community }\end{array}$ \\
\hline
\end{tabular}

Table 1 Summary of the four different activity driven models and their key features

\subsection{SIS epidemic processes unfolding on Model 1: baseline}

As first step, let us consider the basic model where links are created randomly. This is the baseline which will highlight the effects of heterogenous activity patterns (since the link formation is featureless). We can assume that nodes in the same activity class are statistically equivalent. Furthermore, we can differentiate them according to their disease status. Thus we will refer to $S_{a}$, and $I_{a}$ as the number of susceptible and infected nodes in activity class $a$. As we are considering a fixed population $N_{a}=S_{a}+I_{a}$ at all times. In order to derive the conditions for the spreading, we can study the evolution of the infected population. In particular, we can write the number of infected nodes at time $t+\Delta t$ as:

$I_{a}(t+\Delta t)=I_{a}(t)-\mu I_{a}(t) \Delta t+m \lambda S_{a}(t) a \Delta t \int d a^{\prime} \frac{I_{a^{\prime}}(t)}{N}+m \lambda S_{a}(t) \int d a^{\prime} a^{\prime} \Delta t \frac{I_{a^{\prime}}(t)}{N}$.

In particular, this is given by the number of infected nodes at time $t$ (first term on the r.h.s.), minus the nodes that recover (second term on the r.h.s.), plus susceptible nodes that are active, get in contact with infected nodes in other classes and get infected as result (third term on the r.h.s.), plus susceptible nodes that get contacted and infected by active infectious nodes in other categories of activity (fourth term on the r.h.s.). It is important to stress that as each $m$ link is created randomly, the 
probability of selecting a node in a particular class is simply $m / N$. By dividing for $\Delta t$ in the limit of $\Delta t \rightarrow 0$ we can write:

$$
d_{t} I_{a}=-\mu I_{a}+m \lambda \frac{N_{a}-I_{a}}{N} a I+m \lambda \frac{N_{a}-I_{a}}{N} \Theta,
$$

where for simplify the notation we removed the explicit dependence of time, wrote $S_{a}=N_{a}-I_{a}$, defined $\Theta=\int a I_{a} d a$ and considered that $I=\int I_{a} d a$ is the total number of infected nodes. Since we are interested at the early stages of the spreading we can move forward linearizing the expression by assuming that $N_{a} \sim S_{a}$ and by keeping just the first order terms in $I_{a}$. Thus we get

$$
d_{t} I_{a}=-\mu I_{a}+m \lambda \frac{N_{a}}{N} a I+m \lambda \frac{N_{a}}{N} \Theta .
$$

By summing over all classes of activity we get

$$
d_{t} I=-\mu I+m \lambda\langle a\rangle I+m \lambda \Theta,
$$

since $\langle a\rangle=\int a N_{a} / N d a=\int a F(a) d a$. The expression is now function of two variables of $I$ and $\Theta$. In order to understand their behavior we need to get an expression for $\Theta$. To this end, we multiply Eq. 3 by $a$ and sum over all activity classes:

$$
d_{t} \Theta=-\mu \Theta+m \lambda\left\langle a^{2}\right\rangle I+m \lambda\langle a\rangle \Theta .
$$

At this stage, we obtained a system of two differential equations, one in $I$ one in $\Theta$. The disease will be able to spread only if the largest eigenvalue of the Jacobian of the system is larger than zero. In fact, this will imply that the region where we developed the system is unstable. The Jacobian matrix can be written as

$$
J_{m}=\left(\begin{array}{cc}
-\mu+\lambda m\langle a\rangle & \lambda m \\
\lambda m\left\langle a^{2}\right\rangle & -\mu+\lambda m\langle a\rangle
\end{array}\right),
$$

with eigenvalues

$$
\Lambda_{(1,2)}=m\langle a\rangle \lambda-\mu \pm \lambda m \sqrt{\left\langle a^{2}\right\rangle} .
$$

Thus, the epidemic threshold can be simply written as [18]

$$
\frac{\lambda}{\mu}>\frac{1}{m} \frac{1}{\langle a\rangle+\sqrt{\left\langle a^{2}\right\rangle}}
$$

We can define $\beta$ as the per capita rate at which people get infected. This is equal to $\beta=\lambda\langle k\rangle$. The average degree at each time step is equal to $\langle k\rangle=2 m\langle a\rangle$. Thus we can write

$$
\frac{\beta}{\mu}>\xi_{r} \equiv \frac{2\langle a\rangle}{\langle a\rangle+\sqrt{\left\langle a^{2}\right\rangle}},
$$

where we defined $\xi_{r}$ as the epidemic threshold for the random link creation process. It is interesting to notice how the threshold is function of the first and second 
moments of the activity distribution and that it has been derived using also other methods $[38,39,40]$. As the process is unfolding as the network changes structure the threshold is not function of the integrated degree distribution but only of the quantities describing the activation of each node at each time step.

\subsection{SIS epidemic processes in Model 2: the effects of popularity}

Let us now shift gears and analyze the first not random link creation mechanism. In particular, following the previous order, let us consider the global mechanism based on popularity. To this end, nodes are characterized by two features extracted from a general joint distribution $H(a, b)$. As before $a$ describes the activity, $b$ instead the attractiveness. In these settings, it is necessary to divide nodes according to these two features. Thus, the number of susceptible, and infected nodes of activity $a$ and attractiveness $b$ at time $t$ is indicated as $S_{a, b}$ and $I_{a, b}$ respectively. The evolution of the number of infected nodes can be written as:

$$
d_{t} I_{a, b}=-\mu I_{a, b}+\frac{\lambda m}{N\langle b\rangle} S_{a, b}\left[a \iint d a^{\prime} d b^{\prime} b^{\prime} I_{a^{\prime}, b^{\prime}}+b \iint d a^{\prime} d b^{\prime} a^{\prime} I_{a^{\prime}, b^{\prime}}\right] .
$$

The first term on the r.h.s. accounts for the recovery process. The second describes susceptible nodes that are active and select (with probability $b /\langle b\rangle N$ ) infected nodes in other classes getting infected. The third term finally describes susceptible nodes selected by active and infected nodes in other classes and that become infectious as result. It is interesting to notice the symmetry of the last two terms. To move forward, let us define two functions $\theta=\iint a I_{a, b} d a d b$ and $\phi=\iint b I_{a, b} d a d b$. The previous expression becomes:

$$
d_{t} I_{a, b}=-\mu I_{a, b}+\frac{\lambda m}{N\langle b\rangle} S_{a, b}[a \phi+b \theta] .
$$

As before, we can assume that at the early stages of the spreading $N_{a, b} \sim S_{a, b}$ and neglect the terms at the second order in $I_{a, b}$ thus we are left with

$$
d_{t} I_{a, b}=-\mu I_{a, b}+\frac{\lambda m}{N\langle b\rangle} N_{a, b}[a \phi+b \theta] .
$$

From the last expression, we can obtain a system of three equations necessary to study the behavior of the number of infected nodes in the early stages. In particular, we can obtain an expression for i) $I$ by summing all activity and attractiveness classes, ii) $\theta$ by multiplying both sides for $a$ and summing all classes, and iii) $\phi$ by multiplying both sides for $b$ and summing all classes. Doing so, we obtain the following system of differential equations: 


$$
\begin{aligned}
d_{t} I & =-\mu I+\frac{\lambda m}{\langle b\rangle}[\langle a\rangle \phi+\langle b\rangle \theta], \\
d_{t} \theta & =-\mu \theta+\frac{\lambda m}{\langle b\rangle}\left[\left\langle a^{2}\right\rangle \phi+\langle a b\rangle \theta\right], \\
d_{t} \phi & =-\mu \phi+\frac{\lambda m}{\langle b\rangle}\left[\langle a b\rangle \phi+\left\langle b^{2}\right\rangle \theta\right] .
\end{aligned}
$$

The eigenvalues of the Jacobian matrix read:

$$
\Lambda_{1}=-\mu, \quad \Lambda_{(2,3)}=\frac{\lambda m}{\langle b\rangle}\left(\langle a b\rangle \pm \sqrt{\left\langle a^{2}\right\rangle\left\langle b^{2}\right\rangle}\right)-\mu .
$$

As before, the disease is able to spread if the largest eigenvalue is larger than zero. This condition implies [41]:

$$
\frac{\beta}{\mu}>\xi_{a t t} \equiv \frac{2\langle a\rangle\langle b\rangle}{\langle a b\rangle+\sqrt{\left\langle a^{2}\right\rangle\left\langle b^{2}\right\rangle}} .
$$

It is important to notice how the threshold has been computed without any assumption on the form of the distribution $H(a, b)$, thus it is valid for any (integrable) form. A comparison between $\xi_{r}$ and $\xi_{\text {att }}$ reveals how the general structure of the threshold is similar. In particular, the second moments are under the square root in the denominator. However, note how the details of the correlation between the two features appear explicit in the term $\langle a b\rangle$. To gather a deeper understanding on the differences between the two thresholds, let us first consider the uncorrelated case thus $H(a, b)=F(a) G(b)$. In this case, we can re-write the threshold as:

$$
\xi_{a t t}=\frac{2}{1+\sqrt{\frac{\left\langle a^{2}\right\rangle\left\langle b^{2}\right\rangle}{\langle a\rangle^{2}\langle b\rangle^{2}}}} .
$$

As the dependence of the threshold on the two moments is symmetric, the case with constant attractiveness and generic $F(a)$ (baseline) can be mapped to the one with constant activity and attractiveness distribution $F(b)$. Clearly the symmetry would be broken in case of directed networks, since the activity would regulate outlinks while the attractiveness in-links. Furthermore, as $\left\langle b^{2}\right\rangle \geq\langle b\rangle^{2}$ always holds, the threshold can only be lower than or equal to the one found in first model. This means that the introduction of any amount of heterogeneity in the attractiveness helps the epidemic spreading pushing the threshold to smaller values. As mentioned in the introduction, observations in real networks suggest that activity and attractiveness are correlated. The relation between the two, in two online communication networks, can be modeled as $a \sim b^{\gamma_{c}}$ with $\gamma_{c}$ close to one. What happens to the threshold in this case? To answer this question, we can study the case of deterministic correlation between the two variables imposing:

$$
H(a, b)=F(a) \delta(b-q(a)),
$$


where $\delta(x)$ is the Dirac delta and $q(a)$ is the function that determines the attractiveness of a node given its activity: $b_{i}=q\left(a_{i}\right), \forall i$. Using the relation $G(b)=$ $F(a)|d a / d b|$, we can obtain an expression for $G(b)$ :

$$
G(b)=F\left(q^{-1}(b)\right)\left|\frac{d q^{-1}(b)}{d b}\right| .
$$

To account for the observations mentioned above, we can set $q(a)=a^{\gamma_{c}}, \gamma_{c}>0$. Since the activity is distributed according to a power-law $\left(F(a) \propto a^{-\gamma_{a}}\right)$, the attractiveness will be distributed as $G(b) \propto b^{-1+\frac{1-\gamma_{a}}{\gamma_{c}}}$. In these settings, a generic moment of the joint distribution can be expressed as:

$$
\left\langle a^{n} b^{m}\right\rangle=\left\langle a^{n+\gamma_{c} m}\right\rangle
$$

thus the epidemic threshold becomes:

$$
\xi_{a t t}=\frac{2\langle a\rangle\left\langle a^{\gamma_{c}}\right\rangle}{\left\langle a^{\left.1+\gamma_{c}\right\rangle}+\sqrt{\left\langle a^{2}\right\rangle\left\langle a^{2} \gamma_{c}\right\rangle}\right.} .
$$

Generally speaking (this is controlled by the value of $\gamma_{c}$ ) the threshold is not only smaller than $\xi_{r}$ but also smaller than the uncorrelated case. In fact, the disease is able to spread faster when popular people, that are able to attract the connection from many others, are also very active in contacting other nodes.

\subsection{SIS epidemic processes in Model 3: the effects of social memory}

The third model, based on the local reinforcement of previously activated ties (social memory), does not allow (to the best of our knowledge) a derivation of a closed expression for the threshold as we did in the previous two cases. Exact numerical methods, based on the spectral properties of matrices obtained from $G_{t}$, can be used to derive it, but these do not allow to gather an explicit expression [42, 43]. However, a recent paper by Tizzani et al [44] provides an analytical treatment with some approximations. While we refer the interested reader to the original paper for details, here we provide a summary of their derivation as it nicely complements the techniques we discussed above. First of all, they adopted an individual based approach, in which rather than considering classes of activity each node is considered explicitly. In fact, the memory effects for each node make the interactions with a given social circle more likely. Thus nodes in the same activity class cannot be considered statistically equivalent as their behavior depends on their memory of past interactions. In the individual based approach, the focus goes from the study of the evolution of the number of infected nodes in a given activity class to the study of 
the probability $\rho_{i}(t)$ that the node $i$ is infected at time $t$. This can be written as [44]:

$$
\begin{aligned}
& d_{t} \rho_{i}(t)=-\mu \rho_{i}(t)+\lambda\left[1-\rho_{i}(t)\right]\left[\sum_{j} a_{i}\left[1-p\left(k_{i}\right)\right] \frac{A_{i j}(t)}{k_{i}} \rho_{j}(t)+\right. \\
& +\sum_{j{ }^{\prime}} a_{i} p\left(k_{i}\right) \frac{\rho_{j}(t)}{N-k_{i}-1}+\sum_{j} a_{j}\left[1-p\left(k_{j}\right)\right] \frac{A_{i j}(t)}{k_{j}} \rho_{j}(t) \\
& \left.+\sum_{j{ }^{\prime}} a_{j} p\left(k_{j}\right) \frac{\rho_{j}(t)}{N-k_{j}-1}\right]
\end{aligned}
$$

where $A_{i j(t)}$ is the adjacency matrix of the integrated graph up to time $t$, and $\nsim$ selects only the nodes $j$ not yet connected to $i$. By construction this is $N-k_{j}(t)-1$. The first term on the r.h.s. describes the recovery rate of the node. All the other terms describe the infection processes, which depend on the infection probability $\lambda$ and the probability that the node is susceptible $\left(1-\rho_{i}(t)\right)$. The first two terms in the large brackets account for the fact that the node $i$ is active and connects with a node $j$ that has already contacted before (first term) or that has never seen (second term). The last two terms are the same but in this case they account for the fact that the other nodes are active and connect to $i$ [44]. It is important to notice that this expression underlies an approximation: the state of every node is independent of the state of the neighbors. Clearly, this neglects the correlation between nodes. The challenges induced by the memory are clear thinking that the adjacency matrix and the social circle of each node are function of time. Thus, the unfolding of the disease is clearly function of its starting point in time. As Tizzani et al. noted, if we consider the limit in which $1 \ll k_{i}(t) \ll N$, thus if the disease starts spreading when the degree of each node is far from one and from $N$, hence at large times (but not too large), the expression can be reduced as (see Ref. [44] for details):

$$
d_{t} \rho(t)=-\mu \rho(t)+\lambda[1-\rho(t)] \sum_{j} A_{i j}(t)\left(\frac{a_{i}}{k_{i}}+\frac{a_{j}}{k_{j}}\right) \rho_{j}(t) .
$$

In order to find a solution to this expression, Tizzani et al. transitioned from the time integrated connectivity patterns $\left(A_{i j}\right)$ to an annealed form $\left(P_{i j}(t)\right)$ which describes the probability that $i$ and $j$ have been connected in the past. Interestingly, they show that

$$
P_{i j}(t)=(1+\eta) \frac{t^{\frac{1}{1+\eta}}}{N}\left[g\left(a_{i}\right)+g\left(a_{j}\right)\right],
$$

where they defined $g(x)=x /(C x)^{\eta}$. The strength of the memory $\eta$ regulates the expression as well as the activity of the two nodes. From here Tizzani et al can move from the probability that a node $i$ is infected at time $t$ to the probability that a node of activity $a$ at time $t$ is infected (thanks to the annealed approximation). In particular, they obtain [44]: 


$$
\begin{aligned}
& d_{t} \rho(a, t)=-\mu \rho(a, t)+\lambda[1-\rho(a, t)]\left[\frac{a g(a)}{g(a)+\langle g\rangle} \int d a^{\prime} F\left(a^{\prime}\right) \rho\left(a^{\prime}, t\right)\right. \\
& +\frac{a}{g(a)+\langle g\rangle} \int d a^{\prime} F\left(a^{\prime}\right) \rho\left(a^{\prime}, t\right) g\left(a^{\prime}\right)+g(a) \int d a^{\prime} F\left(a^{\prime}\right) \frac{a^{\prime}}{g\left(a^{\prime}\right)+\langle g\rangle} \rho\left(a^{\prime}, t\right) \\
& \left.+\int d a^{\prime} F\left(a^{\prime}\right) \frac{a^{\prime} g\left(a^{\prime}\right)}{g\left(a^{\prime}\right)+\langle g\rangle} \rho\left(a^{\prime}, t\right)\right],
\end{aligned}
$$

where interestingly we find similar terms from the two previous cases. This expression is rather complex. However, the conditions for the spreading can be found, conceptually as before, by linearizing it at early times and studying the Jacobian of the system of four differential equations obtained for it (see Ref. [44] for the derivation). While the condition can be obtained fairly easily numerically, the nature of the terms, and the size of the matrix does not allow for a simple closed expression. Nevertheless, in case a disease start spreading in a mature social network in which nodes have build social circles, this analytical treatment works extremely well [44]. However, the general case, does not have a general closed solution, yet.

\subsection{SIS epidemic processes in Model 4: the effects of communities}

Finally, let us turn our attention to the last model where the link creation dynamic is influenced by the membership to specific communities. In particular, active nodes select (at random) a connection with nodes in their community with probability $q$, and outside their community with probability $1-q$. Although we will not be able to solve them, it is instructive to write the dynamical equations describing the contagion process in these settings. Similarly to what we did before, let us define $S_{a, s}$ and $I_{a, s}$ as the number of susceptible and infected individuals, respectively, in the class of activity $a$ and community of size $s$ at time $t$. We can then write [32]:

$$
\begin{aligned}
d_{t} I_{a, s} & =-\mu I_{a, s}+\lambda a S_{a, s}\left[q \frac{I_{s}}{s}+(1-q) \frac{I}{N}\right] \\
& +\lambda \int d a^{\prime} a^{\prime}\left[q I_{a^{\prime}, s} \frac{S_{a, s}}{s}+(1-q) I_{a^{\prime}, s} \frac{S_{a, s}}{N}\right],
\end{aligned}
$$

where $I_{s}$ and $I$ are the number of infected in communities of size $s$ and in the whole network, respectively. As usual, the first term in the r.h.s accounts for the recovery of infected individuals. The second and third terms describe susceptible nodes that are active and select infected nodes in their community or outside. The fourth and fifth terms are similar but consider that active nodes and infected nodes select susceptible nodes of class $a$ in community of size $s$. For simplicity, we consider that $N-s \sim N$ and, at least initially, $I-I_{s} \sim I$. Summing over all the activities and community sizes, and considering only the first order terms in $a, I_{a, s}$ and their products, we obtain 


$$
\begin{aligned}
d_{t} I & =-\mu I+\lambda\langle a\rangle I+\lambda \theta+\lambda q \sum_{s}\left(\langle a\rangle_{s}-\langle a\rangle\right) I_{s}, \\
d_{t} \theta & =-\mu \theta+\lambda\left\langle a^{2}\right\rangle I+\lambda\langle a\rangle \theta+ \\
& +\lambda q \sum_{s}\left[\left(\left\langle a^{2}\right\rangle_{s}-\left\langle a^{2}\right\rangle\right) I_{s}+\left(\langle a\rangle_{s}-\langle a\rangle\right) \theta_{s}\right],
\end{aligned}
$$

where we defined as before $\theta=\int a I_{a} d a$, and $\theta_{s}=\int a I_{a, s} d a$. The term $\left\langle a^{x}\right\rangle_{s}=$ $\int d a N_{a, s} a^{x} / s$ describes the moments of the activity distribution in any community of size $s$. That is the average activity in a community of size $s$. As before, the second, auxiliary, equation is obtained from the first by multiplying both sides by $a$ and summing over all $s$ and $a$. The epidemic threshold, at least in principle, can be derived evaluating the largest eigenvalue of the Jacobian matrix of the system of differential equations in $I$ and $\theta$. Unfortunately, a closed expression, to the best of our knowledge, has not been derived yet. Nevertheless, we can point out some interesting observations. First of all, the terms in $q$ weight a comparison between the moments of the activity distribution in the whole network with the corresponding values computed inside each community. In case the fluctuations of these terms are negligible, due for example to very large community sizes or to narrow distribution of activity, the equations become equivalent to the case $q=0$ (which is equivalent, for small community sizes, to a random link creation mechanism). Similarly, in case $q \rightarrow 0$, the network has no modular structure, and the threshold becomes equal to the first simple model. In the opposite limit $q \rightarrow 1$ the large majority of connections take place inside each community. Thus the coupling between clusters becomes very weak. Especially when the average size of communities is small, the probability of selecting the same node as partner increases significantly.

Before moving to a more direct comparison between the thresholds in the four different models, let us spend few words about another important, and prototypical, contagion process: the SIR model $[33,34,35]$. While the infection mechanism is equivalent to the SIS, the recovery is radically different. In fact, there is another compartment, $R$, describing infected nodes that recover. These cannot be infected again as they acquire a permanent immunity. It is easy to show that, in case at early stages the population is fully susceptible and thus $R \sim 0$, the threshold for the SIR model unfolding on activity-driven networks with random or global link creation dynamics is equivalent to that of a SIS process. However, the symmetry breaks in case of local link creation mechanisms (for the last model in the limit of high $q$ and small community sizes) [32,45]. In fact, the presence of memory in the connectivity patterns induced by the reinforcement of previously activated ties or by high modularity have opposite effects in SIR and SIS models. The repetition of a small number of connections hinders the spreading power of SIR processes. In fact, as soon as a node recovers, links towards it cannot result in further infections. However, such repetition (as will see more in details in the next section) favors SIS dynamics since it allows the disease to survive in small patches (infected nodes will eventually be susceptible again). 


\section{Epidemic spreading on activity-driven networks: numerical simulations}

In Fig. 2 we show the normalized lifetime, $L_{n}$, for the different variations of the activity-driven framework discussed above. The normalization is done dividing each curve by its maximum, i.e. $L_{n}=L / \max L$. In the plot we considered two versions of the model with heterogenous attractiveness (model 2). In the first, attractiveness and activity, for each node, are extracted independently (uncorrelated scenario). In the second instead, attractiveness and activity are equal (correlated scenario). In the case of social memory (model 3 ), we set $c=1$ and $\eta=1$, thus previously activated ties are repeated with probability $1-p(k)=\frac{k}{1+k}$. We also considered two different versions of the model with communities (model 4). In the first, we set $q=0.45$, thus only $45 \%$ of the links are created within each community. In the second instead, we set $q=0.9$, a much higher value. In both scenarios, community sizes are extracted from a power-law distribution $P(s) s^{-2.1}$ with $10 \leq s \leq \sqrt{N}$. In order to compare the different scenarios, we fixed (across the board) all the other parameters (see legend of Fig. 2 for details). In these settings, several observations are in order. First, all global and local links creation mechanisms result in smaller values of the epidemic threshold respect to the baseline (random links' creation). Second, global, popularity-driven, mechanisms based on heterogenous distributions of attractivity (model 2) push the threshold to much smaller values respect to local mechanisms based on social closeness principles (models 3 and 4). Interestingly, correlations between activity and attractiveness help the spreading of the disease even further respect to uncorrelated scenarios. Third, the effects of communities depend on the value of $q$. In particular, high values of this quantity help the spreading more than smaller values of it. In fact, the repetition of connections within each cluster, helps the disease to survive for smaller values of the spreading rate. Finally, social memory, thus the repetition of previously activated ties, helps the spreading respect to medium-low values of modularity $(q=0.45)$, but not as much as larger values of it. In order to gather a deeper understanding on the dynamics, in Fig. 3 we show, as function of $\beta / \mu$, the fraction of infected population evaluated at the time step equal to the life time, $I_{L}$. This quantity provides complimentary information respect to the previous plot by showing the prevalence of the disease at the moment the conditions that define the lifetime are met (i.e. disease either dies out or reaches a cumulative

fraction $Y$ of the population). Several observations are in order. First, the presence of heterogeneity in nodes' attractiveness (model 2) not only results in the lower value of the threshold (as shown in Fig. 2), but affects a larger fraction of the population respect to all the other links creation mechanisms. The effects of correlations between activity and attractiveness are not as visible as for the threshold. Second, despite in case of strong modularity $(q=0.9)$ the threshold is smaller than in case of social memory, the latter has a larger impact on the population. Indeed, while the presence of tightly connected communities allows for the survival of the disease for smaller values of the spreading rate, high values of modularity confine the impact of the virus in small patches. It is important to notice that this effect is dependent on 


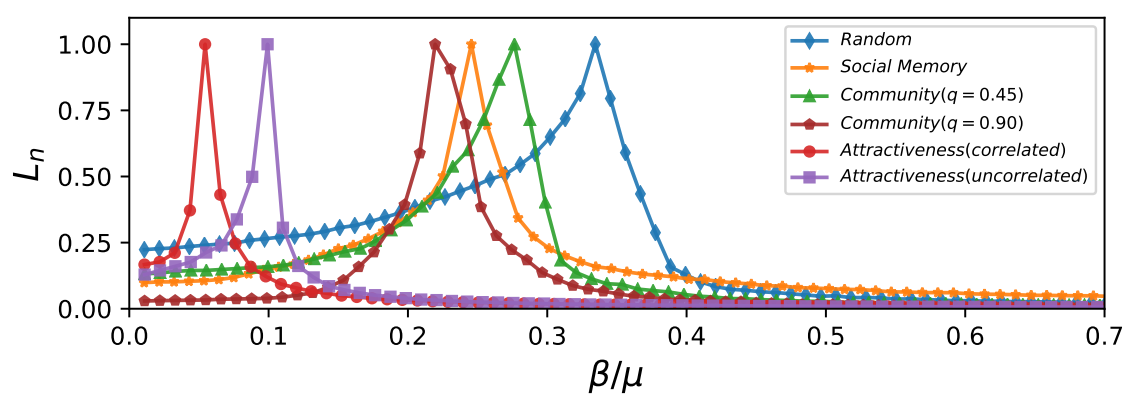

Fig. 2 We show the normalized lifetime of SIS processes unfolding on different variations of the activity driven framework as function of $\beta / \mu$. In all cases, we set $N=10^{5}, m=1, F(a) a^{-2.1}$, $\varepsilon=10^{-3}, \mu=10^{-2}, Y=0.25$, start each simulations with $1 \%$ of randomly selected seeds, and each point is the average of $10^{2}$ independent simulations. In the models with attractiveness, we set the distribution of popularity as $G(b) b^{-2.1}$ and considered two scenarios: 1) $H(a, b)=F(a) G(b)$ (uncorrelated case), 2) $H(a, b)=F(a) \delta(b-a)$ (correlated case). In the model with social memory, we set $c=1$ and $\eta=1$, thus a node that contacted already $k$ nodes will connect to a new tie with probability $p(k)=\frac{1}{k+1}$ and will reinforce a previously activated link with probability $1-p(k)=$ $\frac{k}{k+1}$. Finally, in the models with community structure, we extracted communities sizes for a powerlaw distribution $P(s) s^{-2.1}$ with $10 \leq s \leq \sqrt{N}$ and considered two values of $q$.

the average community size and the distribution of sizes. Intuitively, in case nodes are arranged in few and very large communities such effects would be reduced as the repetition of ties would be much less likely. Finally, social memory (model 3), thus the reinforcement of previously activated ties, has a large impact on the system respect to random and other local mechanisms for values of $\beta / \mu$ closer to the epidemic threshold. As $\beta / \mu$ increases we enter in a region of the phase space where the repetition of the same connections hampers the spreading power of the disease respect to randomly activated ties or to networks with low modularity (model 4). In this regime, the spreading rate is high and having connections with a large number nodes, rather than repeating the links with few of them, helps the unfolding of the disease.

\section{Conclusions}

In this chapter we have investigated the effects of different link creation mechanisms on contagion processes unfolding on time-varying networks. In particular, we focused on two main classes: global and local mechanisms. We modeled the first considering that the propensity of nodes to attract social interactions is heterogeneous. We modeled the second, considering two different approach: i) ties activated in the past are more likely to be re-activated than new ones (social memory), ii) social ties are typically organized in tight communities poorly connected between 


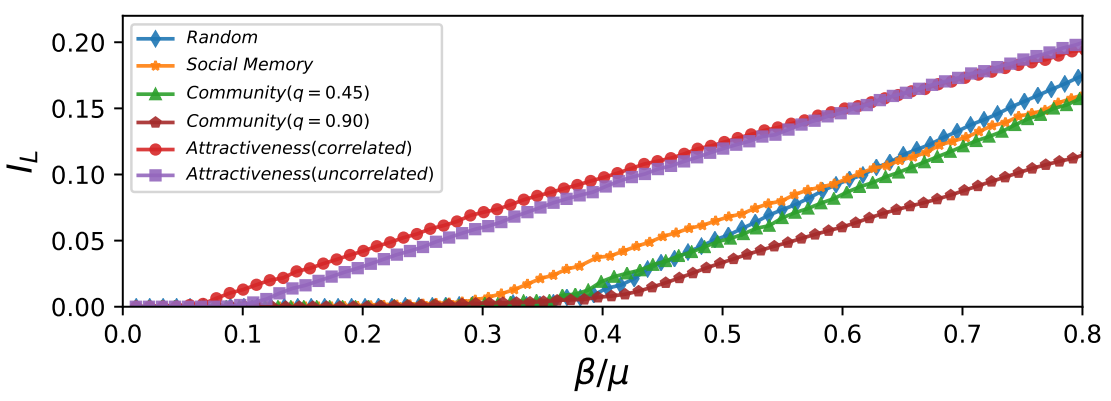

Fig. 3 We show $I_{L}$ for the different version of activity-driven models. This quantity describes the fraction of infected nodes at time $t=L$. The parameters for all different cases are the same used in Fig. 2 .

them. The first mechanism is inspired by popularity (attractiveness) the second by social closeness mechanisms. Furthermore, as null model, we considered the simple, and unrealistic, case in which links are created randomly (baseline). We first provided details about how to analytically tackle the spreading of SIS processes in these models. We then presented a more direct numerical comparison between them. Interestingly, we found that global link creation mechanisms, driven by heterogenous distributions of attractivity, drastically reduce the epidemic threshold respect to the case of homogenous distribution of such quantity (baseline) and to the case of local mechanisms driven by social closeness. Thus, the presence of globally popular nodes, able to attract a large share of the interactions, facilitates the spreading respect to the local correlated dynamics induced by social closeness mechanisms. In fact, as soon such popular hubs get infected they affect a large fraction of the population that connects to them, even for small values of the spreading rate. The effect of communities is function of the modularity. High values of modularity push the threshold to smaller values respect to low values of it and to the social memory mechanism based on the repetition of previously activated ties. However, social memory might have a large impact on system in terms of disease's prevalence in the population. In fact, for values of the spreading rates close to the threshold we observe an interesting phenomenology where the fraction of infected nodes is larger respect to the case of communities as well as to the baseline. While the presence of communities allows the disease to survive for smaller values of the spreading rate, it confines the disease in smaller patches respect to case of social memory.

Arguably, all the mechanisms considered here are not mutually exclusive. In fact, both offline and online social networks are driven by their interplay. Here, we shown that even taken singularly they introduced no-trivial dynamics on contagion processes. More research should be conducted to study their interplay and trade-off in the future. 


\section{References}

1. M. Granovetter. The strength of weak ties. Am. J. Sociol., 78:1360-1380, 1973.

2. Mark Granovetter. Getting a job: A study of contacts and careers. University of Chicago Press, Chicago, 1995.

3. J.-P. Onnela, J. Saramaki, J.and Hyvonen, G. Szabo, D. Lazer, K. Kaski, J. Kertesz, and A.-L. Barabasi. Structure and tie strengths in mobile communication networks. Proc. Natl. Acad. Sci. U.S.A., 104:7332, 2007.

4. Jari Saramäki, Elizabeth A Leicht, Eduardo López, Sam GB Roberts, Felix Reed-Tsochas, and Robin IM Dunbar. Persistence of social signatures in human communication. Proceedings of the National Academy of Sciences, 111(3):942-947, 2014.

5. Eytan Bakshy, Itamar Rosenn, Cameron Marlow, and Lada Adamic. The role of social networks in information diffusion. In Proc. ACM Intl. World Wide Web Conf. (WWW), pages 519-528, 2012.

6. Daniel Z Levin and Rob Cross. The strength of weak ties you can trust: The mediating role of trust in effective knowledge transfer. Management Science, 50(11):1477-1490, 2004.

7. Noah Friedkin. A test of structural features of granovetter's strength of weak ties theory. Social Networks, 2(4):411-422, 1980.

8. Jacqueline Johnson Brown and Peter H. Reingen. Social ties and word-of-mouth referral behavior. Journal of Consumer Research, 14(3):350-362, 1987.

9. S Fortunato. Community detection in graphs. Physics Reports, 486:75-174, 2010.

10. Márton Karsai, Gerardo Iñiguez, Kimmo Kaski, and János Kertész. Complex contagion process in spreading of online innovation. Journal of The Royal Society Interface, 11(101), 2014.

11. Márton Karsai, Mikko Kivelä, Raj Kumar Pan, Kimmo Kaski, János Kertész, A-L Barabási, and Jari Saramäki. Small but slow world: How network topology and burstiness slow down spreading. Physical Review E, 83(2):025102, 2011.

12. Lilian Weng, Márton Karsai, Nicola Perra, Filippo Menczer, and Alessandro Flammini. Attention on weak ties in social and communication networks. In Complex Spreading Phenomena in Social Systems, pages 213-228. Springer, 2018.

13. Ronald S Burt. Structural holes: The social structure of competition. Harvard university press, 2009.

14. M.E.J. Newman. Networks. An Introduction. Oxford Univesity Press, 2010.

15. Albert-László Barabási et al. Network science. Cambridge university press, 2016.

16. Petter Holme. Modern temporal network theory: a colloquium. The European Physical Journal B, 88(9):1-30, 2015

17. Petter Holme and Jari Saramäki. Temporal networks. Physics Reports, 519(3):97-125, 2012.

18. N. Perra, B. Gonçalves, R. Pastor-Satorras, and A. Vespignani. Activity driven modeling of time-varying networks. Scientific Reports, 2:469, 2012.

19. B Ribeiro, N. Perra, and A. Baronchelli. Quantifying the effect of temporal resolution on time-varying networks. Scientific Reports, 3:3006, 2013.

20. M. Karsai, N. Perra, and A. Vespignani. Time varying networks and the weakness of strong ties. Scientific Reports, 4:4001, 2014.

21. M.V. Tomasello, N. Perra, C.J. Tessone, M. Karsai, and F. Schweitzer. The role of endogenous and exogenous mechanisms in the formation of r\&d networks. Scientific Reports, 4:5679, 2014.

22. E. Ubaldi, N. Perra, M. Karsai, A. Vezzani, R. Burioni, and A. Vespignani. Asymptotic theory of time-varying social networks with heterogeneous activity and tie allocation. Scientific Reports, 6:35724, 2016.

23. Laura Alessandretti, Kaiyuan Sun, Andrea Baronchelli, and Nicola Perra. Random walks on activity-driven networks with attractiveness. Physical Review E, 95(5):052318, 2017.

24. Enrico Ubaldi, Alessandro Vezzani, Márton Karsai, Nicola Perra, and Raffaella Burioni. Burstiness and tie activation strategies in time-varying social networks. Scientific reports, $7: 46225,2017$ 
25. Julianne Holt-Lunstad, Timothy B. Smith, and J. Bradley Layton. Social relationships and mortality risk: A meta-analytic review. PLoS Med, 7(7):e1000316, 072010.

26. R. I. M. Dunbar. The social brain hypothesis and its implications for social evolution. Annals of Human Biology, 36(5):562-572, 012009.

27. Giovanna Miritello, Esteban Moro, and Rubén Lara. Dynamical strength of social ties in information spreading. Phys. Rev. E, 83:045102, Apr 2011.

28. James Stiller and R. I. M. Dunbar. Perspective-taking and memory capacity predict social network size. Social Networks, 29(1):93-104, 12007.

29. Joanne Powell, Penelope A. Lewis, Neil Roberts, Marta García-Fiñana, and R. I. M. Dunbar. Orbital prefrontal cortex volume predicts social network size: an imaging study of individual differences in humans. Proceedings of the Royal Society of London B: Biological Sciences, 2012.

30. Bruno Gonçalves, Nicola Perra, and Alessandro Vespignani. Modeling users' activity on twitter networks: Validation of dunbar's number. PloS one, 6(8):e22656, 2011.

31. Guillaume Laurent, Jari Saramäki, and Márton Karsai. From calls to communities: a model for time-varying social networks. The European Physical Journal B, 88(11):1-10, 2015.

32. Matthieu Nadini, Kaiyuan Sun, Enrico Ubaldi, Michele Starnini, Alessandro Rizzo, and Nicola Perra. Epidemic spreading in modular time-varying networks. Scientific Reports, 8(1):2352, 2018.

33. M.J. Keeling and P. Rohani. Modeling Infectious Disease in Humans and Animals. Princeton University Press, 2008.

34. A. Barrat, M. Barthélemy, and A. Vespignani. Dynamical processes on complex networks. Cambridge, 2008.

35. Romualdo Pastor-Satorras, Claudio Castellano, Piet Van Mieghem, and Alessandro Vespignani. Epidemic processes in complex networks. Reviews of Modern Physics, 87(3):925, 2015.

36. Zhen Wang, Chris T Bauch, Samit Bhattacharyya, Alberto d'Onofrio, Piero Manfredi, Matjaž Perc, Nicola Perra, Marcel Salathé, and Dawei Zhao. Statistical physics of vaccination. Physics Reports, 664:1-113, 2016.

37. M. Boguña, C Castellano, and R Pastor-Satorras. Nature of the epidemic threshold for the susceptible-infected-susceptible dynamics in networks. Physical Review Letter, 111:068701, 2013.

38. M. Starnini and R. Pastor-Satorras. Temporal percolation in activity driven networks. Physical Review E, 89:032807, 2014.

39. A. Rizzo, M. Frasca, and M. Porfiri. Effect of individual behavior on epidemic spreading in activity driven networks. Phys. Rev. E, 90:042801, 2014.

40. L. Zino, A. Rizzo, and M. Porfiri. Continuous-time discrete-distribution theory for activitydriven networks. Physical review letters, 117(22):228302, 2016.

41. Iacopo Pozzana, Kaiyuan Sun, and Nicola Perra. Epidemic spreading on activity-driven networks with attractiveness. Physical Review E, 96(4):042310, 2017.

42. Eugenio Valdano, Luca Ferreri, Chiara Poletto, and Vittoria Colizza. Analytical computation of the epidemic threshold on temporal networks. Physical Review X, 5(2):021005, 2015.

43. B.A. Prakash, H. Tong, M. Valler, and C. Faloutsos. Virus propagation on time-varying networks: Theory and immunization algorithms. Machine Learning and Knowledge Discovery in Databases Lecture Notes in Computer Science, 6323:99-114, 2010.

44. Michele Tizzani, Simone Lenti, Enrico Ubaldi, Alessandro Vezzani, Claudio Castellano, and Raffaella Burioni. Epidemic spreading and aging in temporal networks with memory. Physical Review E, 98(6):062315, 2018.

45. Kaiyuan Sun, Andrea Baronchelli, and Nicola Perra. Contrasting effects of strong ties on sir and sis processes in temporal networks. The European Physical Journal B, 88(12):1-8, 2015. 